\title{
Imaging allelic loci in live cells
}

SNP-CLING enables determining the dynamics of alleles on different chromosomes in live cells.

The organization of DNA in the nucleus plays an important role in gene regulation. Yet, methods to study DNA organization often rely on fixing the cells and cross-linking the DNA, preventing researchers from gaining a dynamic view. In particular, distinguishing alleles on different chromosomes is challenging, yet interesting. "The reason this is important is that in most diseases it is only one allele that is mutated," says John Rinn from the University of Colorado at Boulder. $\mathrm{He}$ and his team developed allele-specific CRISPR live-cell imaging (SNP-CLING), which Rinn considers "a logical extension of CRISPR display and CRISPR live-cell imaging... at allele-specific resolution."

For SNP-CLING, the two alleles of interest-harboring single-nucleotide polymorphisms (SNPs) - are targeted by two sets of three single-guide RNAs (sgRNAs), respectively. Each sgRNA contains an RNAaptamer motif in its stem loop adjacent to the guide sequence, which can be bound by a corresponding RNA-binding protein, which in turn is fused to a fluorescent protein. Hence, the two alleles can be labeled with different colors and imaged in live cells. "We can measure individual alleles and their behavior with the relevancy of genetic mutations that can easily be detected, and it can be done in living cells," explains Rinn. This approach is inspired by and extends earlier work on aptamer-based CRISPR-imaging approaches. "We put one and one together and got three," says Rinn.

The team applied their technology to measure the distance of alleles relative to each other and relative to the nucleolus. They found that alleles tend to maintain stable positions over time, and that this distance was not changed during replication.
"The nucleolus is kind of an organizational center. Things seem to be positioned relative to the nucleolus," explains Rinn. However, in cells undergoing apoptosis, the genomic reorganization induced changes in the allelic positioning.

SNP-CLING is not limited to monitoring the behavior of alleles that may have disease implications. Rinn says that the method can also be used for "monitoring how inversions, deletions, insertions move around the genome." He thinks that it could be fun to apply it to watch transposons jump around in the genome. Importantly, the next step will be to monitor allele positioning in differentiating cells to find out whether the chromosome behavior changes.

Nina Vogt

RESEARCH PAPERS

Maass, P.G. et al. Spatiotemporal allele organization by allele-specific CRISPR live-cell imaging (SNPCLING). Nat. Struct. Mol. Biol. 25, 176-184 (2018). 